ECCOMAS

Proceedia
EUROGEN 2021

$14^{\text {th }}$ ECCOMAS Thematic Conference on Evolutionary and Deterministic Methods for Design, Optimization and Control N. Gauger, K. Giannakoglou, M. Papadrakakis, J. Periaux (eds.) Streamed from Athens, Greece, 28-30 June 2021

\title{
PROPELLER OPTIMIZATION STRIVE TO PERFORMANCE / ACOUSTIC TRADE-OFF
}

\author{
Ohad Gur¹, Jonathan Silver², Radovan Dítě ${ }^{3}$, and Raam Sundhar ${ }^{3}$ \\ ${ }^{1}$ Mechanical Design Department \\ ${ }^{2}$ Aerodynamic Department \\ IAI - Israel Aerospace Industries, Lod, 70100, Israel \\ e-mail: \{ogur,jsilver\}@iai.co.il \\ ${ }^{3}$ Aerospace Engineer \\ Mejzlik Propellers s.r.o., Brno-Židenice, 61500, The Czech Republic \\ e-mail: \{dite,sundhar\}@mejzlik.eu
}

\begin{abstract}
This paper describes the design of a propeller-based electric-propulsion system for hover condition. The design procedure harnesses modeFRONTIER optimization framework with various single- and multi-objective hybrid optimization schemes. Several analyses were integrated to the design framework and propeller geometry optimizations were conducted.

The multi-objective problem consisted of trade-off between the contradicting goals of performance (required electric power at hover) and acoustics (tonal overall sound-pressure-level). Using various hybrid optimization schemes, the Pareto tradeoff fronts were found for 2, 3, and 4 bladed propellers. These propellers are compared to an off-the-shelf propeller blade (Mejzlik 18x6) which is used as a reference. This reference propeller proves to be good design, compared to the optimized results. Still, from the optimized Pareto results, 4 propeller configurations were chosen to be fabricated and tested. These configurations are optimized by their acoustic or performance trade-off. These optimized propellers represent a good compromise, which is better than the reference propeller.
\end{abstract}

Keywords: Propeller, Performance, Acoustic, Electric, UAM, Optimization, MDO 


\section{INTRODUCTION}

Urban Air Mobility (UAM) development has been expanding since the publication of UBER-Elevate white paper published in 2016. ${ }^{[1]}$ Since then, numerous manufacturers have been developing various UAM configurations. For example Refs. [2] and [3] show two possible configurations for such vehicles; most of which are multi-propeller based. This makes the propellers a critical item in these vehicles, especially at hover conditions. At hover the propulsion system performance is at its highest required power, ${ }^{[4]}$ thus propeller required-power at hover impacts the overall vehicle performance. In addition, the acoustic signature at hover is the highest and together with new regulations ${ }^{[5]}$ the importance of optimized hovering propellers increases dramatically.

In this paper the design procedure for hover propellers is depicted. The design procedure which includes both analyses, validation, and optimization will be reviewed. From this design process, several propellers were chosen to be fabricated.

Although UAM requires high thrust, an equivalent small propeller is specified, thus the entire design, fabrication and testing procedures are simpler and more rapid. Still, all results are highly related to all hovering configuration, with the appropriate scaling.

This makes the discussed design procedure very useful for future, large scale hoveringpropeller design, especially confronting the complex performance/acoustic tradeoff.

\section{DESIGN SPECIFICATION}

As a reference propeller, the Mejzlik $18 \times 6$ is used. Figure 1 shows the Mejzlik $18 \times 6$ propeller and Figure 2 depicts its geometric properties as function of radial coordinate, $r$, i.e. pitch, $\beta$, chord-to-radius ratio, $c / R$, and thickness-ratio, $t / c$, distribution. A design criterialimits the propeller radius to $R \leq 0.23 \mathrm{~m}$, which is the radius of the reference Mejzlik $18 \times 6$ propeller.

The propeller in the current effort is specified according to its produced thrust. At design conditions, the Mejzlik $18 \times 6$ gives thrust of $T=2.8 \mathrm{kgf}$ which is established as the required thrust for hover (static operation) for all presented designs. The propulsion system is based on Sobek 20-38 Spider brushless DC motor, with Kontrol-X 55LV electronic speed controller, ESC. The acoustic signature is optimized for an observer which is located at azimuth angle, $\theta=100^{\circ}$, relative to the propeller axis, as depicted in Figure 3 . This angle fits the azimuth which generally exhibits the highest sound-pressure-level signature for operated propellers. ${ }^{[6]}$

The above specifications allow the design of propeller with various goals. The most important is the required battery power, electric power, $P_{e}$. Different from other design efforts which refer to the shaft power, here the battery power is the most important, thus the electric propulsion system is to be considered through the design iterations. ${ }^{[7]}$ The second parameter to minimized is the acoustic signature as heard by the observer. This will be considered as the overall sound pressure level, OASPL, at the position of the observer. In the current effort, only the tonal component will be used as design goal. The tradeoff between these two goals is to be found using optimization. 


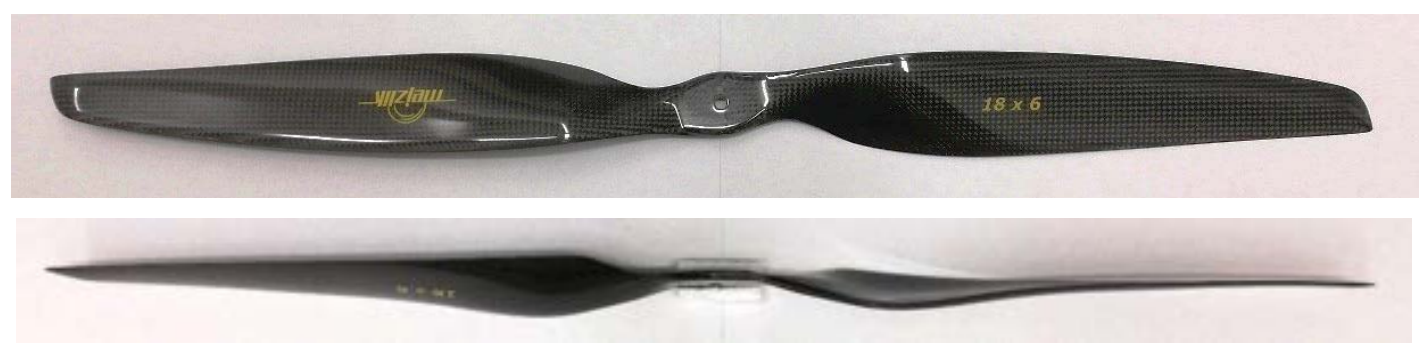

Figure 1: Mejzlik 18×6 Propeller, front and top views

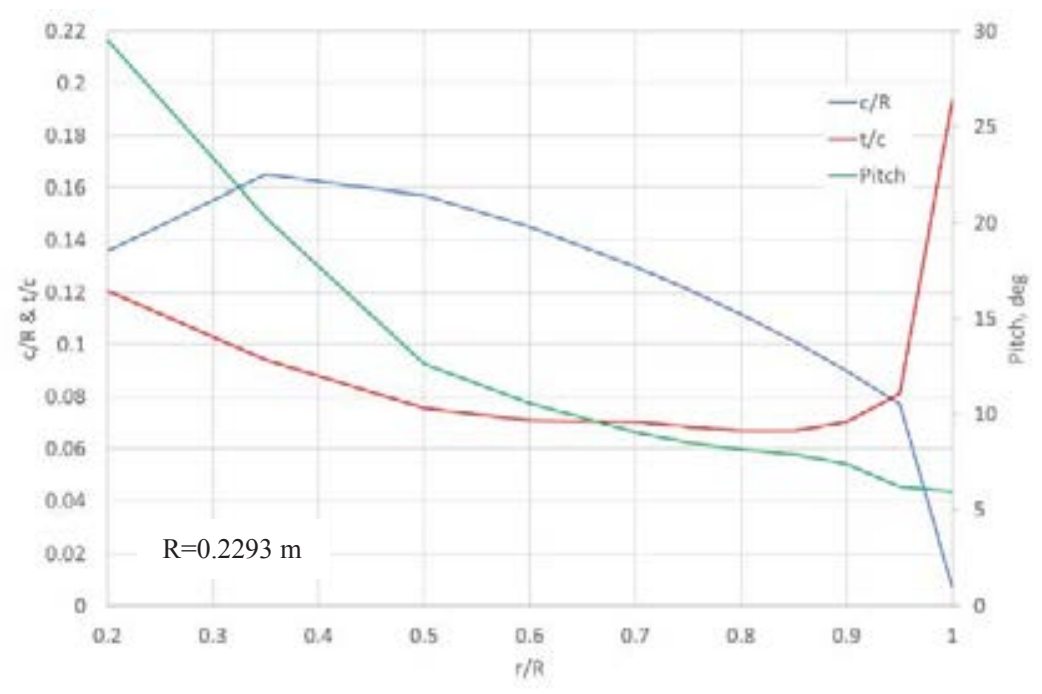

Figure 2: Mejzlik 18×6 Propeller, front and side views

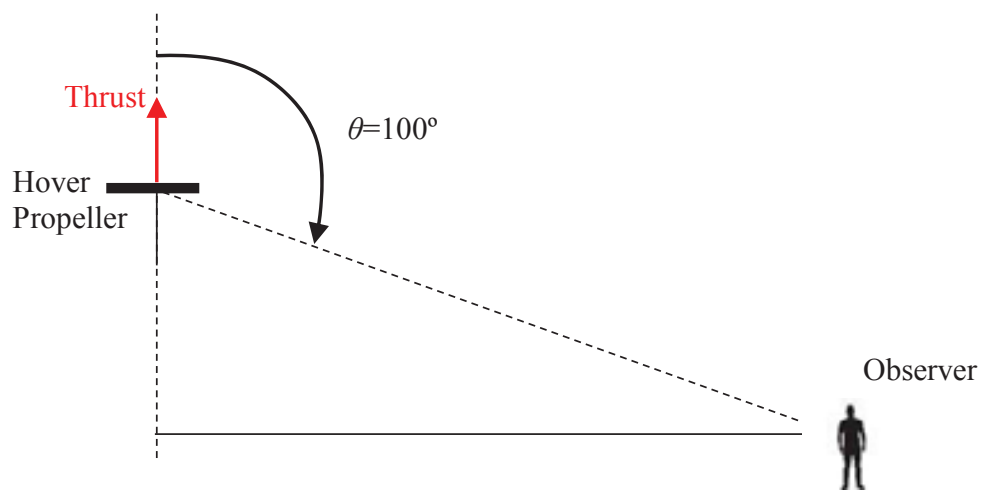

Figure 3: Design conditions of observer-propeller attitude 


\section{PROPELLER'S ANALYSES}

To allow proper optimization, the required analyses should be both accurate and efficient, i.e. using low computer resources. In this case, three analyses are used: propeller performance, electric system, and propeller acoustic analysis.

\subsection{Propeller Performance Analysis}

The propeller performance model is based on blade-element model (BEM) which was extensively validated in the past. ${ }^{[8]}$ Although, most past validation cases were of axial flight regime, in the current case hover condition is treated which was also validated. ${ }^{[7]}$

BEM analysis uses a 2-D aerodynamic database based on the geometry of the propeller cross sectional airfoils. Accuracy of the 2-D aerodynamic database is an important part of BEM level-of-confidence. Thus, substantiation of the current database was conducted using EZair RANS (Reynolds Average Navier-Stokes) software. ${ }^{[9]}$ In addition, some installation losses, due to the propeller and test rig interaction, were implemented on the BEM analysis.

\subsection{Electric System Analysis}

In the current effort a simple motor model is used to find the required electric power. The model is based on four parameters: speed constant, $K_{v}$, armature resistance, $R_{a}$, no-load current, $I_{0}$, and controller efficiency, $\eta_{c}{ }^{[10]}$ The model is based on the following assumptions:

a. Power factor is equal to unit. This assumption is applicable to small brushless Permanent Magnet (PM) motors.

b. Magnetic losses (eddy/Foucault Current and magnetic hysteresis) can be neglected.

\subsection{Acoustic Analysis}

The current acoustic model predicts only the tonal noise of the propeller. The model is based upon Farassat's formulation ${ }^{[11]}$ as used in former design cases ${ }^{[12]}$. The model went through extensive validation for various cases of propeller on various flight regimes. ${ }^{[13],[14]}$

\section{OPTIMIZATION}

Design technique is similar to former cases accomplished with the same tools. These tools include using the validated analysis tools (BEM, electric model, and acoustic model) together with Esteco's modeFRONTIER framework. ${ }^{[13],[7]}$

Figure 4 presents a screen capture of modeFRONTIER framework. This design environment enables the integration of different simulation models into a single synergetic design tool. In addition, it allows the use of various optimization procedures, thus a multidisciplinary design optimization, MDO, tool is obtained. In the current case, first the propeller performance is calculated and then the propeller acoustic is estimated. The use of modeFRONTIER enables an easy usage of any of the input or output parameters, either as design variables or to include it in the goal function and constraints.

In addition, a geometric pre-analysis and performance post-analysis, are implemented using Excel spread sheet. The geometry pre-analysis is used to parametrize the design variables which are the pitch, $\beta$, thickness-ratio, $t / c$, and chord-to-radius ratio, $c / R$, distribution along the blade. The current effort uses a Bezier spline to achieve smooth distribution of the geometry, hence 6 design parameters are used for each distribution. Thus, the design problem contains total of 18 design variables. All airfoils are based on the Mejzlik $18 \times 6$ cross sections, and the propeller radius is fixed to $R=0.23 \mathrm{~m}$. 
To ensure the structural properties of the optimized designs, two geometric constraints are satisfied. First the blade thickness distribution should not be lower than the original Mejzlik $18 \times 6$. Thin blade might be "soft" or exposed to high stresses, which might cause unacceptable aeroelastic behavior, and high deflections. In addition, the root chord should not be larger than the Mejzlik 18×6's root chord. This might cause a very thick hub which increases the propeller weight.

To overcome these issues, the design procedure incorporated two geometric constraints over the thickness distribution and root chord. The first constraint limits the thickness distribution and the second the rood chord. The thickness distribution, $t$ (not $t / c$ ) has to be higher or equal to the Mejzlik $18 \times 6$ up to $r / R=0.90$, with a tolerance of $0.1 \mathrm{~mm}$. The blade tip $(0.9<r / R$ $<1)$ was freed from this constraint - the impact over the design was high and it seems the importance of this constraint, at the very tip of the blade, is less important. The root chord is limited to $c / R<0.15$. Note that the Mejzlik $18 \times 6$ 's $c / R=0.14$ at the root (Figure 2), thus small increase of the root chord is allowed.

The performance post-analysis excel module is used to find the propeller-motor matching speed. Using the performance calculation, for given propeller geometry, several rotational speeds are calculated. To find the correct rotational speed, which the propeller produces the required thrust, $T=2.8 \mathrm{kgf}$, a linear interpolation is used. Then, using the electric model the electric power, $P_{e}$, is found.

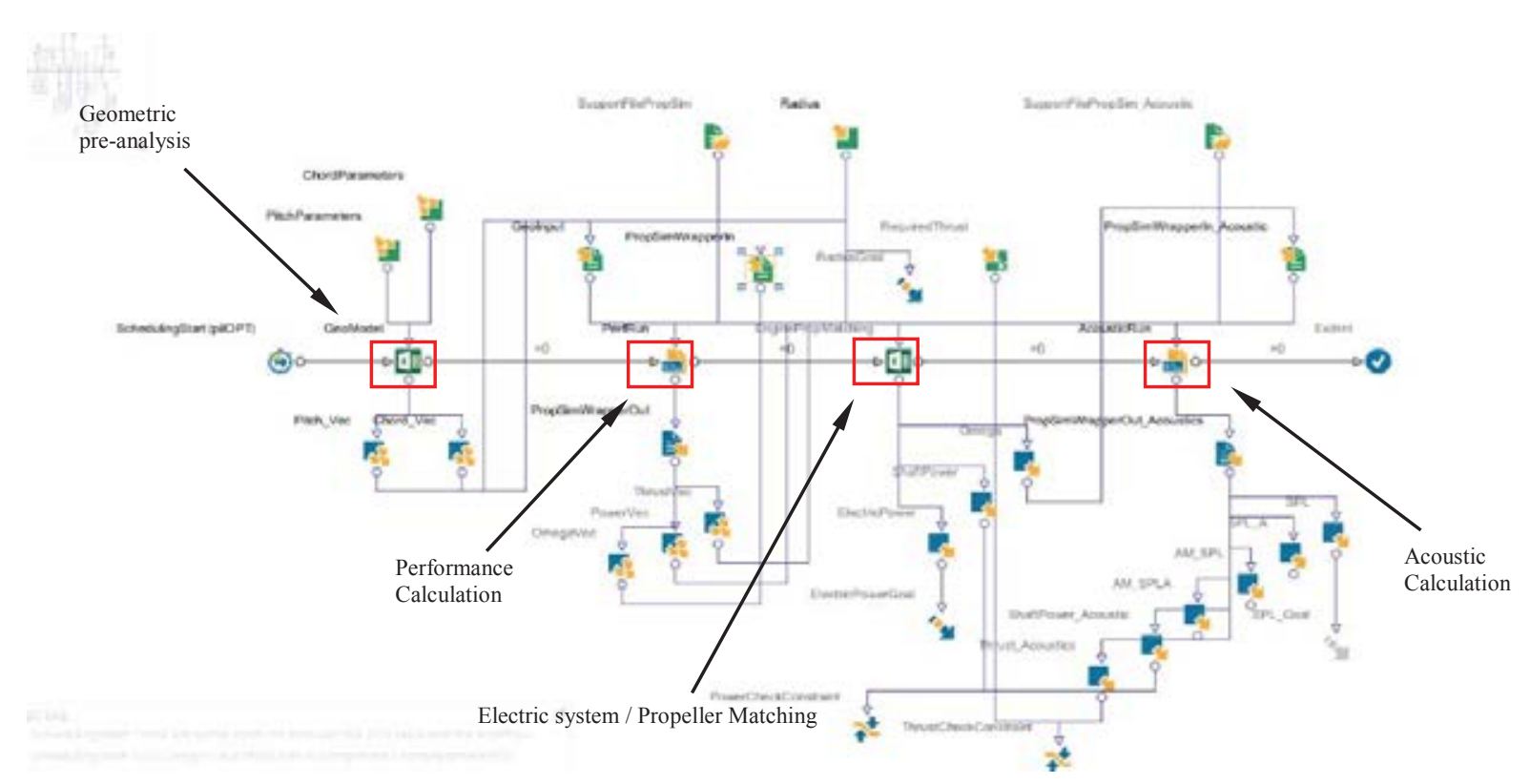

Figure 4: modeFRONTIER design framework screen capture 
Separate optimizations procedures were conducted for 2,3, and 4 bladed propellers. The aim is to find, for different number-of-blades, the tradeoff between the electric power and the overall sound-pressure-level, OASPL, as defined in the problem specification of section 2.

For each specific number-of-blades, the first stage is to find the Utopia Point in the designgoal space. For a multi-objective problem containing two different cost-functions, this is accomplished by two separate single-objective optimizations; the first using the electric power as an objective and the second using the OASPL as the design goal objective. To demonstrate the procedure, the 2-bladed case is considered in what follows.

The single-objective optimizations are conducted using hybrid-optimization scheme based on the available methods in modeFRONTIER. This hybrid scheme can be easily transfer to any other available optimization framework.

First, pilOPT scheme is used. This is highly autonomous method which uses multi-strategy self-adapting algorithm. No design-of-experiment, DOE, is required, nor any other a-priori definition. pilOPT harnesses both surrogate-based (response surface) methods and implicitoptimization methods, thus combines both local and global search techniques.

Using pilOPT, some candidates for further optimization are chosen. These are used as initial guess for constrained gradient-based optimization. In the current effort, sequential quadratic programming, SQP, is used. Each initial guess is optimized into better design; thus a population of optimized solutions are gathered. These are finally used as the initial population for genetic algorithm, GA, scheme, which hopefully finds the global-optimal solution.

Figure 5 shows the results for the two single-objective procedures, conducted for the 2bladed case. The left column is the minimum electric power, $P_{e}$, while the left column is the minim overall sound-pressure-level OASPL. The upper charts show the progress of the costfunction as function of the iteration, Lower charts includes the same results in cost-function space.

Each analysis lasts about $5 \mathrm{sec}$. on a desktop computer using 8 parallel threads of calculation. The entire optimization scheme last about $5 \div 10$ hours, mostly over-night. Some differences between the two cases are visible, mostly for the ratio between the SQP and GA analysis. While the min. $P_{e}$ case uses much more iteration of SQP, the min. OASPL uses more iterations of GA scheme.

In addition, for the min $P_{e}$, the SQP procedure went into a local minimum. Then, the GA scheme "escaped" from this minimum into better region, supposedly global minimum. This is very common for gradient based methods such as SQP to converged to a local minimum. GA is less accurate with its minimum location, but it is capable of hopping to various minimum regions, i.e. global search capabilities. In comparison to the minimum $P_{e}$, the minimum OASPL case exhibits the ability of SQP to locate an optimum which later was improved by the GA scheme. This Hybrid usage of various different scheme, harnesses each scheme's strength to a synergetic optimization procedure.

Note that the designer should carefully monitor the optimization and decide when to move from one method to the other, and which DOE to re-use when transferring from pilOPT to SQP and then from SQP to GA.

Gathering both results of the two single-goal optimization is presented in Figure 6. On this figure also the reference Mejzlik 18x6 propeller is presented as a green circle. The cloud of results can be used to substantiate DOE which is then used to optimize the Pareto frontier. This is done using a multi-goal scheme, in the current case mainly by MOGA and NSGA schemes. The final Pareto frontier after optimization is presented as a black curve in Figure 6 . Note that the cloud of results from the two single-objective optimizations, draw the final Pareto with relatively good accuracy. Thus, the Utopia-point estimation, actually plays an important role for the multi-objective scheme. 

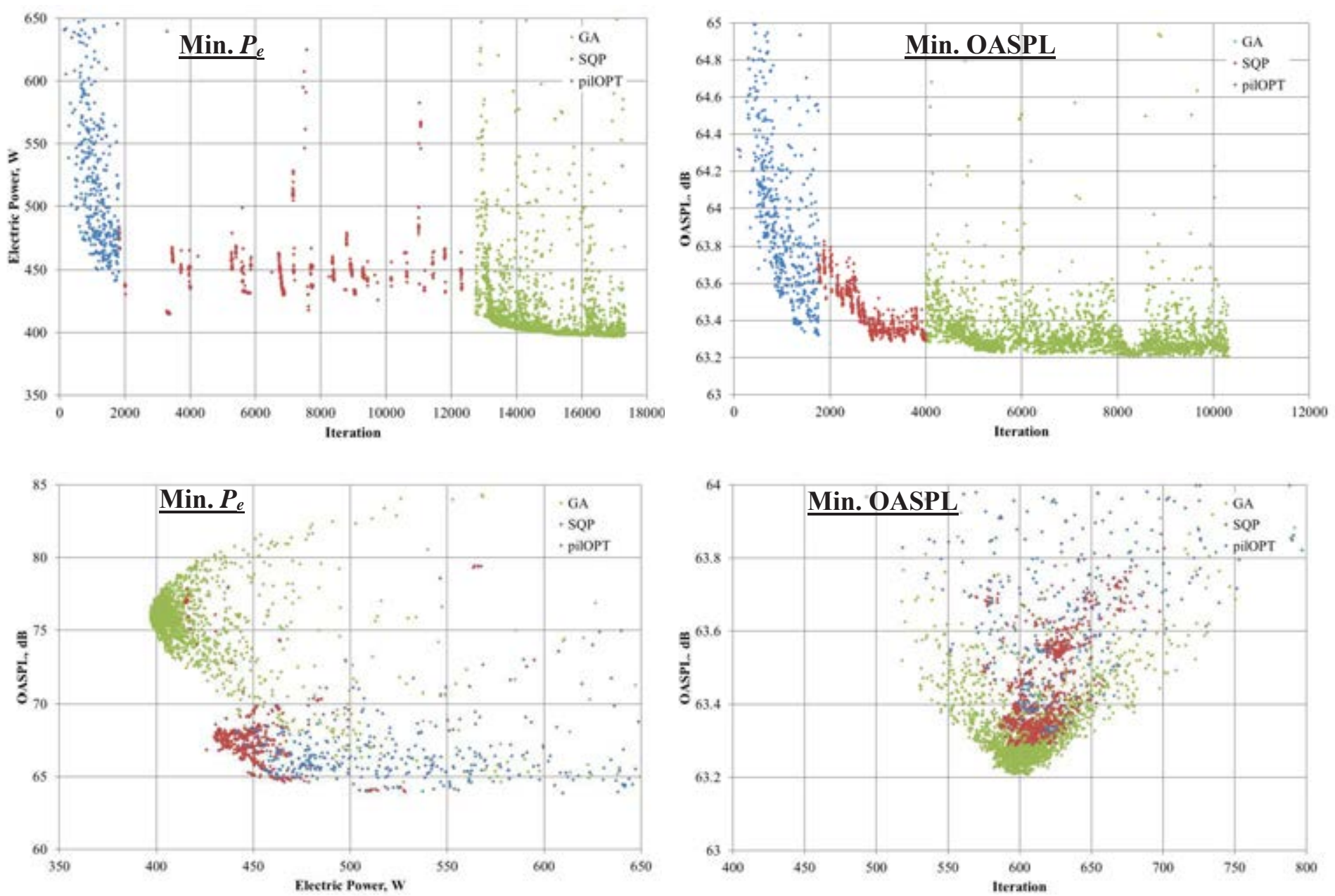

Figure 5: 2-blades Utopia Point, single-objective optimization results Left: minimum electric power, $P_{e}$, Right: minimum overall sound-pressure-level OASPL

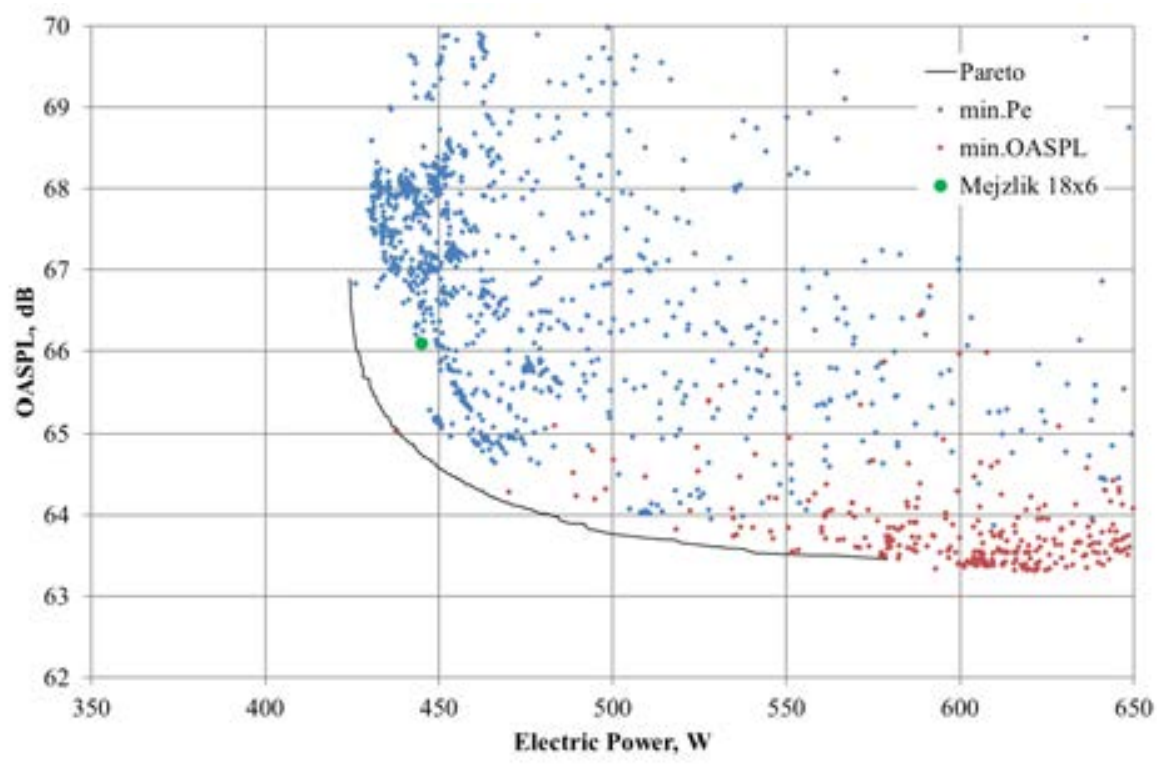

Figure 6: 2-blades single-objective results compared to the reference Mejzlik 18x6 and final Pareto frontier 
The multi-goal optimization was conducted 3 times for 2, 3, and 4 blades configurations. Each uses the same procedure mentioned above. Pareto frontiers which resulted from the optimization are presented in fig. 7. Propellers based on the Mejzlik $18 \times 6$ blades are also marked by red circles. The design influence of number-of-blades is prominent - increased number-of-blades causes both OASPL decrease and $P_{e}$ increase - a tradeoff which the designer should consider carefully.

From these Pareto frontiers, 4 propeller configurations were chosen - these are marked with arrows in fig. 7 and include:

a. 2 blades, minimum $P_{e}\left(2 \mathrm{~B} \min P_{e}\right)$

b. 2 blades, minimum SPL (2B min SPL)

c. 3 blades, minimum $P_{e}\left(3 \mathrm{~B} \min P_{e}\right)$

d. 4 blades, minimum $P_{e}\left(4 \mathrm{~B} \min P_{e}\right)$

These selections are based on motivation for improving the already adequate Mejzlik 18x6, on different aspects. The minimum $P_{e}$ is being chosen as improved $P_{e}$ without penalizing the OASPL. Similarly, min. OASPL is chosen with no penalty over $P_{e}$.

The propeller characteristics are depicted in table 1, and their blade geometric parameters in fig.8. The clear difference is the rotational speed. This appears both as the mechanism of reducing the OASPL for the 2 blades propeller and for achieving the proper thrust for the 3 and 4 bladed propeller. To reduce the rotational speed, thus achieving min SPL for the 2 bladed propeller, the pitch was increased and the chord slightly increased as well.

For the 3 and 4 blades, the rotational speed had to decrease to achieve the required thrust, $T=2.8 \mathrm{kgf}$. The chord cannot decrease due to the geometric constraint, thus the chord remained similar and thickness remains above the Mejzlik $18 \times 6$ blade. To maintain high enough rotational speed, the pitch decreases for the 3 and 4 bladed propellers, thus the electric efficiency, $\eta_{e}$, and figure-of-merit, FM, remain relatively high.

While the 3-bladed propeller exhibits high FM and low $\eta_{e}$, the 4-bladed exhibits low FM and high $\eta_{e}$. Generally, all tradeoff in such complex design case, is beyond simple intuition and it is a result of handling with all constraints while striving to minimize all design goals. This proves the advantage of such MDO (multidisciplinary design optimization) framework, which takes contradicting requirements and find the best compromise. 


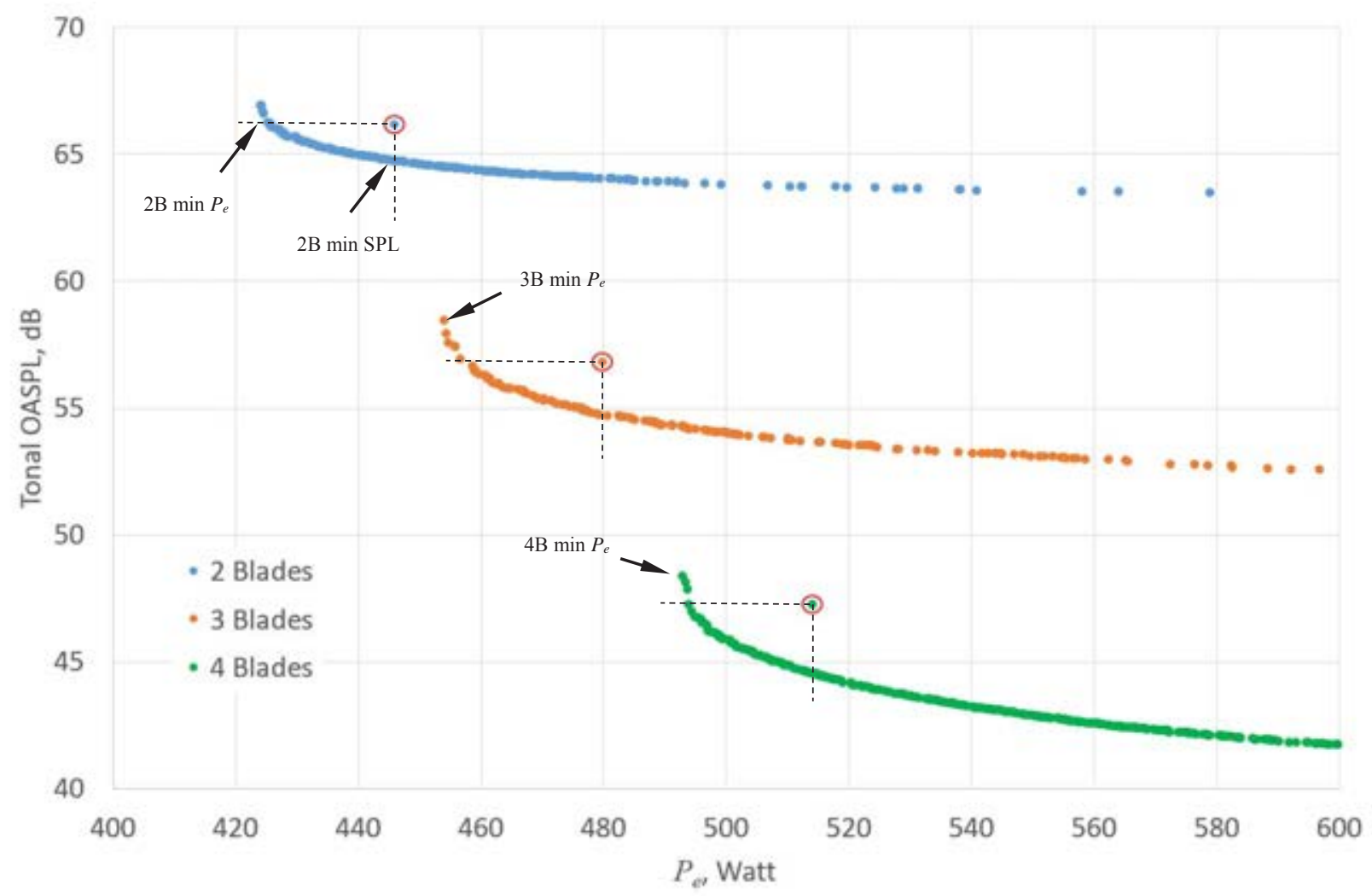

Figure 7: Pareto frontiers for the optimized results

Red circles mark the results for propeller based on Mejzlik $18 \times 6$ blades

\begin{tabular}{|l|c|c|c|c|c|}
\hline & $\begin{array}{c}\text { 2-Blades } \\
\text { Mejzlik 18 } \times 6\end{array}$ & $\begin{array}{c}\text { 2-Blades } \\
\text { min. } P_{e}\end{array}$ & $\begin{array}{c}\text { 2-Blades } \\
\text { min.SPL }\end{array}$ & $\begin{array}{c}\text { 3-Blades } \\
\text { min. } P_{e}\end{array}$ & $\begin{array}{c}\text { 4-Blades } \\
\text { min. } P_{e}\end{array}$ \\
\hline Electric Power, $P_{e}, \mathrm{~W}$ & 445 & 425 & 445 & 455 & 495 \\
\hline Shaft Power, $P_{\text {shaft, }} \mathrm{W}$ & 340 & 335 & 345 & 350 & 375 \\
\hline Engine Speed, $\Omega, \mathrm{rpm}$ & 5,100 & 5,200 & 4,600 & 4,700 & 4,500 \\
\hline Figure-of-merit, FM & 0.68 & 0.69 & 0.67 & 0.66 & 0.62 \\
\hline Electric efficiency, $\eta_{e}$ & 0.77 & 0.79 & 0.77 & 0.67 & 0.76 \\
\hline Tonal OASPL, dB & 66.1 & 66.1 & 64.7 & 56.9 & 48.3 \\
\hline
\end{tabular}

Table 1: Optimized propeller characteristics 

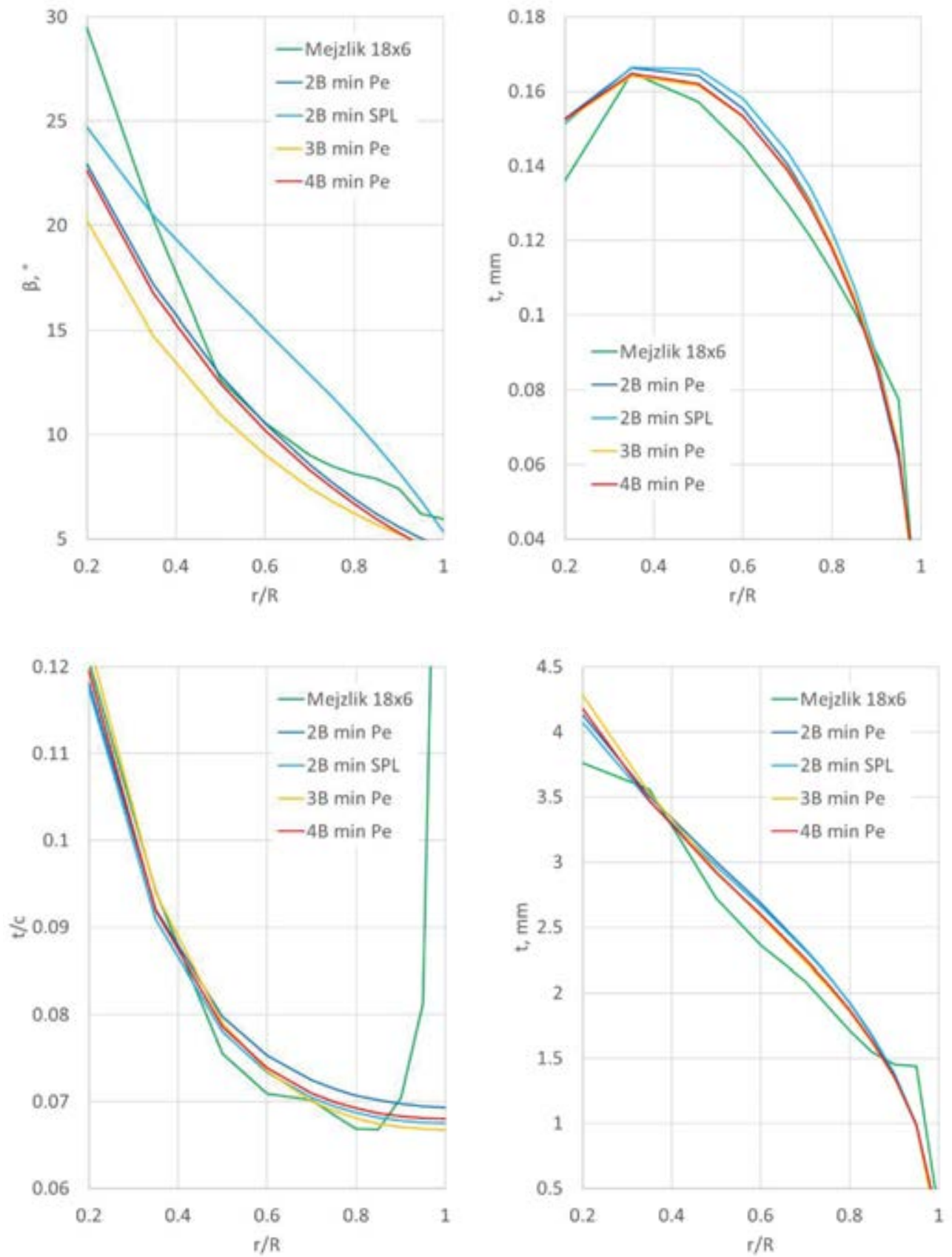

Figure 8: Optimized blade geometries 


\section{CONCLUSIONS}

In this paper a comprehensive and methodic design process for hover-propeller is described. The design process has to have a detailed specification which is based, in the current case, on an existing propulsion system with of-the-shelf propeller. In the basis of the design process are 3 analytic models: blade-element model for the propeller performance estimation, electric model for the propulsion system characteristics, and acoustic model which analyzes the propeller tonal sound-pressure-level. Each of these models was previously validated versus various results in the literature.

These analyses were incorporated in a design framework based on modeFRONTIER software and a multidisciplinary-design-optimization environment was substantiated. This environment includes, beside the analyses, various definitions of design variables, constraints, and design goals. Hence a multi-objective optimization problem is defined.

The design framework was run 3 times for designing 2, 3, and 4 bladed propellers. First, a Utopia-point was found using a single-goal optimization process, which resulted with mature design-of-experiment for the final multi-goal scheme. The optimization harnesses various schemes such as multi-strategy, gradient-based, and evolutionary.

The optimization scheme was resulted with a Pareto frontier which exhibits the tradeoff between the propulsion-system performance and its acoustic signature. From these tradeoffs, optimized propeller configurations were chosen. These are to be fabricated and tested. The test results for both performance and acoustics is to be compared with the design trends, thus the design process is to be validated.

In the current effort 4 propeller were resulted. Two of them are 2 bladed, minimal electric power and minimal acoustic signature. In addition, 3 bladed and 4 bladed propellers for minimum electric power were chosen. The four propellers exhibited some improvements over the reference of-the-shelf propeller. These improvements can be chosen by the designer according to the resulted Pareto frontiers. This demonstrates the use of Pareto tradeoff results as a quantitative, important decision support tool, during design process.

\section{ACKNOWLEDGMENTS}

The research was funded by Israel-Europe Research \& Innovation Directorate, ISERD, of the Israel Innovation Authority, and DELTA-2 programme of the Technology Agency of the Czech Republic, TAČR. The authors thank these two organizations for their generous contributions. 


\section{REFERENCES}

[1] UBER Elevate, "Fast-Forwarding to a Future of On-Demand Urban Air Transportation," October 2016 [URL: https://www.uber.com/il/he/elevate/ Cited: September 2020]

[2] S. Rogers, "This Passenger Drone Is Set to Have Its First Test Flight Next Week," January 2018 [URL: https://interestingengineering.com/this-passenger-drone-is-set-tohave-its-first-test-flight-next-week Cited: September 2020]

[3] A. Douglas, "Honeywell and Volocopter sign navigation solutions R\&D deal," Commercial Drone Professional April 2019 [URL: https://www.commercialdroneprofessional.com/honeywell-and-volocopter-signnavigation-solutions-rd-deal/ Cited: September 2020]

[4] Gur O, Lazar G., and Yaniv A., "Electric VTOL UAV prop-rotor MDO," 52nd Israel Annual Conference on Aerospace Sciences, February 29 - March 1st, 2012, Israel

[5] EASA, "COMMISSION DELEGATED REGULATION (EU) 2019/945, of 12 March 2019 on unmanned aircraft systems and on third-country operators of unmanned aircraft systems," Official Journal of the European Union, March 2019 [URL: https://eurlex.europa.eu/legal-content/EN/TXT/PDF/?uri=CELEX:32019R0945\&from=EN Cited: September 2020]

[6] The Royal Aeronautical Society, "Estimation of the maximum discrete frequency noise from isolated rotors and propellers," ESDU 76020, February 2018

[7] Gur O, Lazar G., "Prop-rotor design for an electric tilt-rotor vehicle," American Helicopter Society, Future Vertical Lift Aircraft Design Conference, January 18-20, 2012, Fisherman's Wharf, San Francisco, California

[8] Gur O. and Rosen A., "Comparison between blade-element models of propellers", 48th Israel Annual Conference on Aerospace Sciences, February 27-28, 2008, Israel.

[9] Y. Kidron, Y. Levy, and M. Wasserman, "The EZAir CFD Solvers Suite: EZAir Theoretical and User's Manual - Version 3.54,” Tech. Rep. August, ISCFDC, 2016.

[10] Gur O. and Rosen A., "Optimization of electric UAV propulsion system," Journal of Aircraft, Vol. 46, No. 4, July-August 2009, pp. 1340-1353

[11] Farassat, F., and Succi, G. P., "A Review of Propeller Discrete Frequency Noise Prediction Technology with Emphasis on Two Current Methods for Time Domain Calculations," Journal f Sound and Vibration, Vol. 71, No. 3, 1980, pp. 399-419

[12] Gur O. and Rosen A., "Design of quiet propeller for an electric mini unmanned air vehicle," Journal of Power and Propulsion, Vol. 25, No. 3, May-June 2009, pp.717-728

[13] Gur O. and Rosen A., "Optimization of Propeller Based Propulsion System," AIAA Journal of Aircraft, Vol. 46, No. 1, January-February 2009, pp. 95-106

[14] Gur O., "Toward Optimal Design of Contra-Rotating Propulsion System - Practical Acoustic Analysis," 1st Aerospace Europe Conference AEC2020 Bordeaux, France, 25-28 February 2020 